\title{
Analysis and Implementation of Cohen_Sutherland Line Clipping Algorithm
}

\author{
Honglin Li \\ School of Information Engineering, Qujing Normal University, Qujing Yunnan 655011 \\ email:lihonglin28286@sina.com
}

Keywords: Cohen_Sutherland; Improve; Line; Clip

\begin{abstract}
In this paper,through the analysis of the advantages and disadvantages of Cohen_Sutherland linear cutting algorithm, and the improved algorithms are studied, the improved algorithms can be better and more comprehensive to determine the straight line in the window.
\end{abstract}

\section{Introduction}

Clipping is the basis of computer graphics, image processing and other subjects. Because the efficiency of clipping has a direct impact on the efficiency of computer graphics, image processing and so on, so it is of great significance to improve the efficiency of clipping[1].

Clipping is a kind of operation to cut out the window of an object [2]. It's most basic purpose is to determine the designated area within a scene, this area is called the window, clipping is to define the selected area in this window. It's principle is to display a window of a large picture of the local content, in accordance with the contents of the window boundary conditions can be displayed, and does not meet the content required to cut [3].

In the use of computer graphics information processing, often appear inside the computer graphics is relatively large, and the screen can only be displayed as part of the map. At this time, the graph should be cut. At present, the common line clipping algorithm has Cohen_Sutherland coding algorithm, the midpoint segmentation algorithm, Nicholl-Lee-Nicholl algorithm[4][5] , Liang_Barsky algorithm[6], Andreev-Sofianska [7], and so on. In this paper, we mainly study the Cohen_Sutherland line clipping algorithm, and the improved algorithm is analyzed and compared.

\section{Cohen_Sutherland Line Clipping}

The Basic Idea of the Cohen_Sutherland Algorithm. Cohen-Sutherland clipping algorithm is also known as coding clipping method[8]. The basic idea of the algorithm is that:

(1) If the line segments of the two end points of the code all zero, namely two end points of a line segment coding logical bitwise OR operation, if the "or" operation result is zero, line completely with in the window ,we should be reserved it, namely "take", otherwise, turn to (2).

(2)If the two end points of a line of code with a 1, the two ends of the line of code for according to a logic "and" operation, if the result is not zero, line completely outside of the window, and we should be discarded, that is disposable, otherwise, turn to (3).

(3) Straight line and window may intersect.we need to get the intersection coordinates of the line segment, then the line is segmented in two, respectively to deal with (1) and (2), until all Lines are divided into two segments, either fully retained or completely discarded,so far,the algorithm is end.

Shortcomings of Cohen_Sutherland Algorithm. There are two possibilities for the line segment completely outside of the window.(1) The two end points of the line segment are encoded with a 1, that is the two end points of the line segment are on the same side of the window.(2) The window is located on the same side of the line segment, but the two end points of the line segment are not identical with one bit at the same time, and the two end points of the line segment are encoded according to the bit logic "and" operation, the result is zero. The second case, Cohen_Sutherland algorithm can not quickly determine its completely outside of the window, we need to hand in hand with the window, the results found that the intersection is invalid, resulting in the algorithm to deal with such line is lower efficiency. At the same time, if 
the line is not satisfied with the complete reservation, also does not meet the completely discarded, the line segment should to get intersection coordinates with the boundary of the window, and the calculation involves the multiplication and division of floating point, the algorithm efficiency is not high.

\section{Improved Cohen_Sutherland Algorithm}

Currently, many methods of improving the Cohen_sutherland algorithm, much of the improvement are is to complete a straight line outside the window. The line segment's two end points code without a binary number is 1 at the same time, in order to avoid unnecessary intersection computing,we had improved it, in the following,there are three kinds of improved algorithm as an example.

(1)First improved methods .Firstly, the two end points of cut line segment are coded. And then carry out the following tests[1].

(1) The two end points of the line segment encoding logic or operation, if the result is 0000 , the line segment is completely in the window, we can be completely retained.

(2)Logical and operation of the two end points of the line segment, if the result is true (nonzero), the line is completely outside of the window, you can completely abandon.

(3) If it does not satisfy (1) and (2), the two vertex above the window substituted into the equation of the straight line, if the result is less than zero at the same time, indicating that line completely on top of windows, completely abandoning. Then, the two vertex below of the rectangular window substituted into the equation of the straight line, if the result is greater than zero at the same time, indicating that the line completely under the window, completely abandoning.

For the first, second, and third, are not satisfied, just need to make the calculation, this kind of line segment must have a hand with the window edge.

(2)Cond improved methods. Firstly, the two endpoints of cut line segment are coded. And then carry out the following tests[9].

(1) The two end points of the line segment are encoded and then to make logic or operation, if the result is 0000 , the line completely is in the window, completely retained.

(2) The two end points of the region codes are to make logic and operation, if the result is true (non-zero), the line is completely outside the window, completely abandoned.

(3) The window of the four vertices put into linear equations, if the symbol is the same as that the line is complete in the window, you can abandon it completely.

For the line segment are not satisfied with the above situations, need to make the calculation, these lines must pass through inside of the window.

The above two algorithms, although for completely outside the window of the second case is by adding a judgment condition, making all completely in line outside the window can quickly filter, to avoid seeking intersection point about the boundary of the window, we need to let the window each vertex coordinates into the linear equation to judge whether the line is completely outside the window, there are also floating point multiplication and division operations. The method is more efficient when most of the line segments do not need to be cut, and the efficiency of the algorithm is reduced if the majority of the line segments to be cut are not completely preserved and completely discarded.

(3)Third improved methods.In view of the Cohen_Sutherland algorithm, the straight line which is completely outside the window can not be judged effectively. We can use directed distance method[10]. Known a rectangular window, the lower left corner of the coordinates (xleft, ybottom), the upper right corner of the coordinates (xright, ytop), a line is to be cut out which of the two coordinates of the line is $\left(\mathrm{x}_{1}, \mathrm{y}_{1}\right),\left(\mathrm{x}_{2}, \mathrm{y}_{2}\right)$. The linear equation is: $A x+B y+C=0$, which: $\mathrm{A}=\mathrm{y} 2-\mathrm{y} 1 ; \mathrm{B}=\mathrm{x} 2-\mathrm{x} 1 ; \mathrm{C}=\mathrm{x} 2 * \mathrm{y} 1-\mathrm{x} 1 * \mathrm{y} 2$. A point $(\mathrm{x}, \mathrm{y})$ to a straight line's distance is $\mathrm{d}$, then 


$$
d=\frac{A \times x+B \times y+C}{\sqrt{A^{2}+B^{2}}} .
$$

Assuming that the window of the vertices coordinates as fllows: (xleft, ytop), (xleft, ybottom), (xright, ytop), (xright, ybottom), and each vertex which distance to be cutting a straight line's distance as follows: $\mathrm{d} 1, \mathrm{~d} 2, \mathrm{~d} 3, \mathrm{~d} 4$. if $\mathrm{d} 1$ and $\mathrm{d} 2$ have the same symbol , or $\mathrm{d} 3$ and $\mathrm{d} 4$ have the same symbol,we can just that the straight line is out of the window, we can directly discard it . If d1 and $\mathrm{d} 2$ have the diversity symbol, or $\mathrm{d} 3$ and $\mathrm{d} 4$ have the diversity symbol, the line may intersect to the window, and then we can use the traditional Cohen_Sutherland algorithm to calculate the intersection point coordinate.

This method is by adding a judgment condition, making all the line completely outside of the window can quickly filter out, to avoid to calculate the intersection point coordinate of the line which intersects the boundary of the window, but we calculate the distance of the line to the vetices of of the window, this process is still time consuming.

\section{Comparison of Algorithms}

The traditional Cohen_Sutherland algorithm, improved Cohen_Sutherland algorithm (1), (2), and (3), we compare various algorithms, as shown in Table 1.

Table 1 Algorithm Running Time [s]

\begin{tabular}{|l|c|c|c|c|}
\hline & $\begin{array}{c}\text { Cohen- } \\
\text { Sutherland }\end{array}$ & $\begin{array}{c}\text { Improved } \\
\text { Cohen_Sutherla } \\
\text { nd algorithm(1) }\end{array}$ & $\begin{array}{c}\text { Improved } \\
\text { Cohen_Sutherla } \\
\text { nd algorithm(2) }\end{array}$ & $\begin{array}{c}\text { Improved } \\
\text { Cohen_Sutherla } \\
\text { nd algorithm(3) }\end{array}$ \\
\hline A straight line & 12.944886 & 12.765567 & 11.822642 & 11.208981 \\
(cutting & 12.919162 & 12.031527 & 11.807352 & 11.367368 \\
50 million) & 12.897874 & 12.733332 & 11.877882 & 11.315567 \\
\hline 8Straight & 10.590412 & 10.575306 & 10.562123 & 10.218263 \\
Lines (cutting & 10.731081 & 10.574696 & 10.429169 & 10.141270 \\
5 million) & 11.169227 & 10.443266 & 10.526579 & 10.186438 \\
\hline
\end{tabular}

From the table we can know as the third improved method, which is directed distance is faster. In short, the efficiency of these improved algorithms's efficiency is not very obvious.

\section{Conclusion}

In this paper, we introduce the shortcomings of the Cohen-Sutherland algorithm, and then we improve and analyze it, and we are using computer programming. It has a certain practical value.

\section{References}

[1] H.P. Wang. Research and improvement of clipping algorithm for two dimensional graphics(Ph.D, Xi'an Electronic and Science University, China 2011).P.18. (In Chinese)

[2] H.X. Yina, S.CH. Du and LJ Cai:Computer Graphics(China water conservancy and Hydropower Press, China 2005),P.70. (In Chinese)

[3] R. Wang.Circular clipping algorithm based on rectangle window coding(Ph.D,Liaoning Normal University, China 2011). (In Chinese)

[4] Nicholltm,Leedt and Nichollra:Computer Graphics, Vol,21(1987)No.4,p.253-262. (In Chinese) 
[5] Y.D. Liang,B.A. Barsky:ACM,Vol,3(1984)No.1,P.1-22.

[6] J.G. Sun:Computer graphics(Tsinghua university press,China 1998),p.200-206. (In Chinese)

[7] Andreevr and sofianskaf:Computer \& Graphics, Vol.15(1991)No.4,p.519-526.

[8] Ko Imanic S, Guid N. Journal of Computing and Information Technology, (2003) No.4, p.319-326.

[9] Y.J. Wang, G.Q. Xiao and H.H. Ren:Modern computer, Vol,2(2007),p.15-16. (In Chinese)

[10] J.J. Guo,ZH.ZH. Yang:Science and technology innovation herald, (2015), No.18,p. 47. (In Chinese). 